\title{
Positivity, Betweenness, and Strictness of Operator Means
}

\author{
Pattrawut Chansangiam \\ Department of Mathematics, Faculty of Science, King Mongkut's Institute of Technology Ladkrabang, Bangkok 10520, Thailand
}

Correspondence should be addressed to Pattrawut Chansangiam; kcpattra@kmitl.ac.th

Received 5 March 2015; Accepted 7 May 2015

Academic Editor: Sergei V. Pereverzyev

Copyright (C) 2015 Pattrawut Chansangiam. This is an open access article distributed under the Creative Commons Attribution License, which permits unrestricted use, distribution, and reproduction in any medium, provided the original work is properly cited.

An operator mean is a binary operation assigned to each pair of positive operators satisfying monotonicity, continuity from above, the transformer inequality, and the fixed-point property. It is well known that there are one-to-one correspondences between operator means, operator monotone functions, and Borel measures. In this paper, we provide various characterizations for the concepts of positivity, betweenness, and strictness of operator means in terms of operator inequalities, operator monotone functions, Borel measures, and certain operator equations.

\section{Introduction}

The concept of means, a natural notion in mathematics, plays important roles in mathematics itself, computer science, statistics, various branches in science, engineering, and economics. This concept was developed since the ancient Greeks until the last century by many mathematicians (see [1]). Nowadays, according to the definition of a mean for positive real numbers in [1], a mean $M$ is defined to be satisfied by the following properties:

(i) Positivity: $s>0$ and $t>0 \Rightarrow M(s, t)>0$.

(ii) Betweenness: $s \leqslant t \Rightarrow s \leqslant M(s, t) \leqslant t$.

A mean $M$ is said to be

(i) strict at the left if for each $a>0$ and $b>0$,

$$
M(a, b)=a \Rightarrow a=b
$$

(ii) strict at the right if for each $a>0$ and $b>0$,

$$
M(a, b)=b \Rightarrow a=b
$$

(iii) strict if it is strict at both the right and the left.

This paper focuses on means for positive operators on a Hilbert space. Let $B(\mathscr{H})$ be the algebra of bounded linear operators on a Hilbert space $\mathscr{H}$. The set of positive operators on $\mathscr{H}$ is denoted by $B(\mathscr{H})^{+}$. Denote the spectrum of an operator $X$ by $\operatorname{Sp}(X)$. For self-adjoint operators, $A, B \in B(\mathscr{H})$, the partial order $A \leqslant B$ indicates that $B-A \in B(\mathscr{H})^{+}$. If $A \in B(\mathscr{H})^{+}$is invertible, then we write $A>0$.

A starting point for the theory of operator means is the presence of the notion of parallel sum in electrical network analysis (see [2]). A connection is a binary operation $\sigma$ assigned to each pair of operators in $B(\mathscr{H})^{+}$such that the following conditions are satisfied for all $A, B, C, D \in B(\mathscr{H})^{+}$:

(M1) monotonicity: $A \leqslant C, B \leqslant D \Rightarrow A \sigma B \leqslant C \sigma D$;

(M2) transformer inequality: $C(A \sigma B) C \leqslant(C A C) \sigma(C B C)$;

(M3) continuity from above: for $A_{n}, B_{n} \in B(\mathscr{H})^{+}$, if $A_{n} \downarrow A$ and $B_{n} \downarrow B$, then $A_{n} \sigma B_{n} \downarrow A \sigma B$. Here, $X_{n} \downarrow X$ indicates that $X_{n}$ is a decreasing sequence (with respect to the partial order) and $X_{n}$ converges strongly to $X$.

This definition was modelled from significant properties of the parallel sum by Kubo and Ando in [3]. Two trivial examples are the left-trivial mean $\omega_{l}:(A, B) \mapsto A$ and the right-trivial mean $\omega_{r}:(A, B) \mapsto B$. See [4, Section 3] and [5] for more information about operator connections. From the transformer inequality, every connection is congruence invariant in the sense that for each $A, B \geqslant 0$ and $C>0$ we have

$$
C(A \sigma B) C=(C A C) \sigma(C B C) .
$$


A mean in Kubo-Ando sense is a connection $\sigma$ with fixedpoint property $A \sigma A=A$ for all $A \geqslant 0$. The class of KuboAndo means cover many well-known means in practice, for example,

(i) $\alpha$-weighted arithmetic means: $A \nabla_{\alpha} B=(1-\alpha) A+\alpha B$;

(ii) $\alpha$-weighted geometric means:

$$
A \#_{\alpha} B=A^{1 / 2}\left(A^{-1 / 2} B A^{-1 / 2}\right)^{\alpha} A^{1 / 2}
$$

(iii) $\alpha$-weighted harmonic means:

$$
A !_{\alpha} B=\left[(1-\alpha) A^{-1}+\alpha B^{-1}\right]^{-1}
$$

(iv) logarithmic mean: $(A, B) \mapsto A^{1 / 2} f\left(A^{-1 / 2} B A^{-1 / 2}\right) A^{1 / 2}$ where the function $f$ is given by $f(x)=(x-1) / \log x$ for each $x \in \mathbb{R}^{+} \equiv[0, \infty), f(0) \equiv 0$, and $f(1) \equiv 1$.

A summary of Kubo-Ando theory is given in terms of one-to-one correspondences between operator connections on $B(\mathscr{H})^{+}$, operator monotone functions from $\mathbb{R}^{+}$to $\mathbb{R}^{+}$, and finite Borel measures on $[0,1]$. Recall that a continuous function $f: \mathbb{R}^{+} \rightarrow \mathbb{R}^{+}$is said to be operator monotone if

$$
A \leqslant B \Longrightarrow f(A) \leqslant f(B)
$$

for all positive operators $A, B \in B(\mathscr{H})$ and for all Hilbert spaces $\mathscr{H}$. This concept was introduced in [6]; see also [7, Chapter V], [4, Section 2], and [8]. A connection $\sigma$ on $B(\mathscr{H})^{+}$ can be characterized via operator monotone functions as follows.

Theorem 1 (see [3, Theorem 3.2]). Given a connection $\sigma$, there is a unique operator monotone function $f: \mathbb{R}^{+} \rightarrow \mathbb{R}^{+}$satisfying

$$
f(x) I=I \sigma(x I), \quad x \geqslant 0 .
$$

Moreover, the map $\sigma \mapsto f$ is a bijection.

We call $f$ the representing function of $\sigma$. A connection also has a canonical characterization with respect to a Borel measure via a meaningful integral representation as follows.

Theorem 2. Given a finite Borel measure $\mu$ on $[0,1]$, the binary operation

$$
A \sigma B=\int_{[0,1]} A !_{t} B d \mu(t), \quad A, B \geqslant 0
$$

is a connection on $B(\mathscr{H})^{+}$. Moreover, the map $\mu \mapsto \sigma$ is bijective, in which case the representing function of $\sigma$ is given by

$$
f(x)=\int_{[0,1]}\left(1 !_{t} x\right) d \mu(t), \quad x \geqslant 0 .
$$

Theorem 2 is a modification of Kubo-Ando theorem ([3, Theorem 3.4]). We call $\mu$ the associated measure of $\sigma$.
Theorem 3 (see [3, Theorem 3.3]). Let $\sigma$ be a connection on $B(\mathscr{H})^{+}$with representing function $f$ and associated measure $\mu$. Then the following statements are equivalent:

(1) $I \sigma I=I$;

(2) $A \sigma A=A$ for all $A \in B(\mathscr{H})^{+}$;

(3) $f$ is normalized; that is, $f(1)=1$;

(4) $\mu$ is normalized; that is, $\mu$ is a probability measure.

Hence every mean can be regarded as an average of weighted harmonic means. From (8) and (9) in Theorem 2, $\sigma$ and $f$ are related by

$$
f(A)=I \sigma A, \quad A \geqslant 0 .
$$

In this paper, we provide various characterizations for the concepts of positivity, betweenness, and strictness of operator means in terms of operator inequalities, operator monotone functions, Borel measures, and certain operator equations. It turns out that every mean satisfies the positivity property. The betweenness is a necessary and sufficient condition for a connection to be a mean. A mean is strict at the left (right) if and only if it is not the left-trivial mean (the right-trivial mean, resp.).

\section{Positivity}

We say that a connection $\sigma$ satisfies the positivity property if

$$
A>0, \quad B>0 \Longrightarrow A \sigma B>0 .
$$

Recall that the transpose of a connection $\sigma$ is the connection

$$
(A, B) \longmapsto B \sigma A
$$

If $f$ is the representing function of $\sigma$, then the representing function of its transpose is given by

$$
g(x)=x f\left(\frac{1}{x}\right), \quad x>0,
$$

and $g(0)$ is defined by continuity (see [3, Corollary 4.2]).

Theorem 4. Let $\sigma$ be a connection on $B(\mathscr{H})^{+}$with representing function $f$ and associated measure $\mu$. Then the following statements are equivalent:

(1) $\sigma$ satisfies the positivity property;

(2) $I \sigma I>0$;

(3) $\sigma \neq 0$ (here, 0 is the zero connection $(A, B) \mapsto 0$ );

(4) for all $A \geqslant 0, A \sigma A=0 \Rightarrow A=0$ (positive definiteness);

(5) for all $A \geqslant 0, A \sigma I=0 \Rightarrow A=0$;

(6) for all $A \geqslant 0, I \sigma A=0 \Rightarrow A=0$;

(7) for all $A \geqslant 0$ and $B>0, A \sigma B=0 \Rightarrow A=0$;

(8) for all $A>0$ and $B \geqslant 0, A \sigma B=0 \Rightarrow B=0$;

(9) $f \neq 0$ (here, 0 is the function $x \mapsto 0$ );

(10) $x>0 \Rightarrow f(x)>0$;

(11) $\mu([0,1])>0$. 
Proof. Implications (1) $\Rightarrow(2) \Rightarrow(3),(4) \Rightarrow$ (3), (7) $\Rightarrow$ $(5) \Rightarrow(3)$, (8) $\Rightarrow$ (6) $\Rightarrow$ (3), and (10) $\Rightarrow$ (9) are clear. Using the integral representations in Theorem 2 , it is straightforward to verify that the representing function of the zero connection $0:(A, B) \mapsto 0$ is the constant function $f \equiv 0$ and its associated measure is the zero measure. Hence, we have the equivalences (3) $\Leftrightarrow$ (9) $\Leftrightarrow$ (11).

$(9) \Rightarrow(10)$. Assume $f \neq 0$. Suppose that there is $a>0$ such that $f(a)=0$. Then $f(x)=0$ for all $x \leqslant a$. The concavity of $f$ implies that $f(x)=0$ for all $x \geqslant a$. Hence $f=0$, a contradiction.

$(5) \Rightarrow(7)$. Assume (5). Let $A \geqslant 0$ and $B>0$ be such that $A \sigma B=0$. Then

$$
0=B^{1 / 2}\left(B^{-1 / 2} A B^{-1 / 2} \sigma I\right) B^{1 / 2}
$$

and $B^{-1 / 2} A B^{-1 / 2} \sigma I=0$. Now, (5) yields $B^{-1 / 2} A B^{-1 / 2}=0$; that is, $A=0$.

$(6) \Rightarrow(8)$. It is similar to $(5) \Rightarrow(7)$.

$(10) \Rightarrow(1)$. Assume that $f(x)>0$ for all $x>0$. Since $\operatorname{Sp}(f(A))=f(\operatorname{Sp}(A))$ by spectral mapping theorem, we have $f(A)>0$ for all $A>0$. Hence, for each $A>0$ and $B>0$,

$$
A \sigma B=A^{1 / 2} f\left(A^{-1 / 2} B A^{-1 / 2}\right) A^{1 / 2}>0 .
$$

$(10) \Rightarrow(4)$. Assume (10). Let $A \geqslant 0$ be such that $A \sigma A=0$. Note that

$$
\begin{aligned}
A \sigma A & =\lim _{\epsilon \downarrow 0} A_{\epsilon} \sigma A_{\epsilon}=\lim _{\epsilon \downarrow 0} A_{\epsilon}^{1 / 2}(I \sigma I) A_{\epsilon}^{1 / 2} \\
& =\lim _{\epsilon \downarrow 0} f(1) A_{\epsilon}=f(1) A ;
\end{aligned}
$$

here $A_{\epsilon} \equiv A+\epsilon I$. Since $f(1)>0$, we have $A=0$.

$(10) \Rightarrow(5)$. Assume (10). Let $A \geqslant 0$ be such that $A \sigma I=0$. Then $g(A)=0$ where $g$ is the representing function of the transpose of $\sigma$. We see that $g(x)>0$ for $x>0$. The injectivity of functional calculus implies that $g(\lambda)=0$ for all $\lambda \in \operatorname{Sp}(A)$. We conclude that $\operatorname{Sp}(A)=0$; that is, $A=0$.

$(10) \Rightarrow(6)$. Assume (10). Let $A \geqslant 0$ be such that $I \sigma A=0$. Then $f(A)=0$. By the injectivity of functional calculus, we have $f(\lambda)=0$ for all $\lambda \in \mathrm{Sp}(A)$. Assumption (10) implies that $\operatorname{Sp}(A)=\{0\}$. Thus, $A=0$.

Remark 5. It is not true that $\sigma \neq 0$ implies the condition that for all $A \geqslant 0$ and $B \geqslant 0, A \sigma B=0$ implies $A=0$ or $B=0$. Indeed, take $\sigma$ to be the geometric mean and

$$
\begin{aligned}
& A=\left(\begin{array}{ll}
1 & 0 \\
0 & 0
\end{array}\right), \\
& B=\left(\begin{array}{ll}
0 & 0 \\
0 & 1
\end{array}\right) .
\end{aligned}
$$

\section{Betweenness}

We say that a connection $\sigma$ satisfies the betweenness property if for each $A \geqslant 0$ and $B \geqslant 0$,

$$
A \leqslant B \Longrightarrow A \leqslant A \sigma B \leqslant B
$$

By Theorem 4, every mean enjoys the positivity property. In fact, the betweenness property is a necessary and sufficient condition for a connection to be a mean.

Theorem 6. The following statements are equivalent for a connection $\sigma$ with representing function $f$ :

(1) $\sigma$ is a mean;

(2) $\sigma$ satisfies the betweenness property;

(3) for all $A \geqslant 0, A \leqslant I \Rightarrow A \leqslant A \sigma I \leqslant I$;

(4) for all $A \geqslant 0, I \leqslant A \Rightarrow I \leqslant I \sigma A \leqslant A$;

(5) for all $t \geqslant 0,1 \leqslant t \Rightarrow 1 \leqslant f(t) \leqslant t$;

(6) for all $t \geqslant 0, t \leqslant 1 \Rightarrow t \leqslant f(t) \leqslant 1$;

(7) for all $A \geqslant 0$ and $B \geqslant 0, A \leqslant B \Rightarrow\|A\| \leqslant\|A \sigma B\| \leqslant$ $\|B\|$;

(8) for all $A \geqslant 0, A \leqslant I \Rightarrow\|A\| \leqslant\|A \sigma I\| \leqslant 1$;

(9) for all $A \geqslant 0, I \leqslant A \Rightarrow 1 \leqslant\|I \sigma A\| \leqslant\|A\|$;

(10) the only solution $X>0$ to the equation $X \sigma X=I$ is $X=I$;

(11) for all $A>0$, the only solution $X>0$ to the equation $X \sigma X=A$ is $X=A$.

Proof. Implications (2) $\Rightarrow$ (3), (2) $\Rightarrow$ (4), (2) $\Rightarrow(7) \Rightarrow$ (8), and $(11) \Rightarrow(10) \Rightarrow(1)$ are clear.

$(1) \Rightarrow(2)$. Let $A, B \geqslant 0$ be such that $A \leqslant B$. The fixedpoint property and the monotonicity of $\sigma$ yield

$$
A=A \sigma A \leqslant A \sigma B \leqslant B \sigma B=B .
$$

$(3) \Rightarrow(1)$. Since $I \leqslant I$, we have $I \leqslant I \sigma I \leqslant I$; that is, $I \sigma I=I$. Hence $\sigma$ is a mean by Theorem 3 .

$(4) \Rightarrow(1)$. It is similar to $(3) \Rightarrow(1)$.

$(8) \Rightarrow(1)$. We have $1=\|I\| \leqslant\|I \sigma I\| \leqslant 1$. Hence,

$$
f(1)=\|f(1) I\|=\|I \sigma I\|=1 .
$$

Therefore, $\sigma$ is a mean by Theorem 3 .

$(7) \Rightarrow(9) \Rightarrow(1)$. It is similar to $(7) \Rightarrow(8) \Rightarrow(1)$.

$(1) \Rightarrow(11)$. Let $A>0$. Consider $X>0$ such that $X \sigma X=$ $A$. Then by the congruence invariance of $\sigma$, we have

$$
X=X^{1 / 2}(I \sigma I) X^{1 / 2}=X \sigma X=A .
$$

$(2) \Rightarrow(5)$. If $t \geqslant 1$, then $I \leqslant I \sigma(t I) \leqslant t I$ which is $I \leqslant$ $f(t) I \leqslant t I$; that is, $1 \leqslant f(t) \leqslant t$.

$(5) \Rightarrow(1)$. We have $f(1)=1$.

$(2) \Rightarrow(6) \Rightarrow(1)$. It is similar to $(2) \Rightarrow(5) \Rightarrow(1)$.

Remark 7. For a connection $\sigma$ and $A, B \geqslant 0$, the operators $A, B$ and $A \sigma B$ need not be comparable. The previous theorem tells us that if $\sigma$ is a mean, then the condition $0 \leqslant A \leqslant B$ guarantees the comparability between $A, B$ and $A \sigma B$.

\section{Strictness}

We consider the strictness of Kubo-Ando means as that for scalar means in [1]. 
Definition 8. A mean $\sigma$ on $B(\mathscr{H})^{+}$is said to be

(i) strict at the left if for each $A>0$ and $B>0$,

$$
A \sigma B=A \Rightarrow A=B
$$

(ii) strict at the right if for each $A>0$ and $B>0$,

$$
A \sigma B=B \Rightarrow A=B
$$

(iii) strict if it is strict at both the right and the left.

In order to prove the next two lemmas, recall the following facts: if $f: \mathbb{R}^{+} \rightarrow \mathbb{R}^{+}$is operator monotone, then

(i) $f$ is operator concave and hence concave in usual sense (see [9] or [4, Corollary 2.5.4]);

(ii) $g(x)=x f(x)$ is convex in usual sense (see [3, Lemma $5.2])$;

(iii) $h(x)=x / f(x)$ is operator monotone on $(0, \infty)$ (see [9] or [4, Corollary 2.5.6]).

Lemma 9. If $f: \mathbb{R}^{+} \rightarrow \mathbb{R}^{+}$is an operator monotone function such that $f$ is a constant on an interval $[a, b]$ with $a<b$, then $f$ is a constant on $\mathbb{R}^{+}$.

Proof. Assume that $f(x)=k$ for all $a \leqslant x \leqslant b$. The case $a=0$ is done by using the monotonicity and concavity of $f$. Consider the case $a>0$. The monotonicity and concavity of $f$ imply that $f(x)=k$ for all $x \geqslant b$. If $k=0$, then $f(x)=k$ on $[0, a]$ by the monotonicity of $f$. Consider the case $k>0$ and suppose there is an $x_{0} \in[0, a)$ such that $f\left(x_{0}\right)<k$. Then the slope of the line segment joining the point $\left(x_{0}, x_{0} f\left(x_{0}\right)\right)$ and the point $(a, a f(a))$ is greater than $k$. This contradicts the convexity of the function $x f(x)$.

Lemma 10. If $f: \mathbb{R}^{+} \rightarrow \mathbb{R}^{+}$is an operator monotone function such that $f(x)=m x+c$ for some $m>0$ and $c \geqslant 0$ on an interval $[a, b]$ with $a<b$, then $f(x)=m x+c$ on $\mathbb{R}^{+}$.

Proof. If there is $x_{0}>0$ such that $f\left(x_{0}\right)=0$, then $f \equiv 0$ by Lemma 9. Suppose that $f(x)>0$ for all $x>0$. For simplicity, assume that $f(x)=x$ for all $a \leqslant x \leqslant b$. Then the function $h(x)=x / f(x)$ is operator monotone on $(0, \infty)$ and hence on $\mathbb{R}^{+}$by continuity. Note that $h(x)=1$ on $[a, b]$. Lemma 9 implies that $h(x)=1$ on $\mathbb{R}^{+}$; that is, $f(x)=x$ on $\mathbb{R}^{+}$.

Theorem 11. Let $\sigma$ be a mean with representing function $f$ and associated measure $\mu$. Then the following statements are equivalent:

(1) $\sigma$ is strict at the left;

(2) $\sigma$ is not the left-trivial mean;

(3) for all $A \geqslant 0, I \sigma A=I \Rightarrow A=I$;

(4) for all $A>0, A \sigma I=A \Rightarrow A=I$;

(5) for all $A>0$ and $B \geqslant 0, A \sigma B=A \Rightarrow A=B$;

(6) for all $A \geqslant 0, I \leqslant I \sigma A \Rightarrow I \leqslant A$;

(7) for all $A \geqslant 0, I \sigma A \leqslant I \Rightarrow A \leqslant I$;
(8) for all $A>0, A \leqslant A \sigma I \Rightarrow A \leqslant I$;

(9) for all $A>0, A \sigma I \leqslant A \Rightarrow I \leqslant A$;

(10) for all $A>0$ and $B \geqslant 0, A \leqslant A \sigma B \Rightarrow A \leqslant B$;

(11) for all $A>0$ and $B \geqslant 0, A \sigma B \leqslant A \Rightarrow B \leqslant A$;

(12) $f$ is not the constant function $x \mapsto 1$;

(13) for all $x \geqslant 0, f(x)=1 \Rightarrow x=1$;

(14) for all $x \geqslant 0, f(x) \geqslant 1 \Rightarrow x \geqslant 1$;

(15) for all $x \geqslant 0, f(x) \leqslant 1 \Rightarrow x \leqslant 1$;

(16) $\mu$ is not the Dirac measure at 0 .

Proof. It is clear that (5) $\Rightarrow$ (1) and each of (1), (4) and (6)-(11) implies (2). Also, each of (13)-(15) implies (12).

$(2) \Rightarrow(3)$. Let $A \geqslant 0$ be such that $I \sigma A=I$. Then $f(A)=I$ and hence $f(\lambda)=1$ for all $\lambda \in \operatorname{Sp}(A)$. Suppose that $\alpha \equiv$ $\inf \operatorname{Sp}(A)<r(A)$ where $r(A)$ is the spectral radius of $A$. Then $f(x)=1$ for all $x \in[\alpha, r(A)]$. It follows that $f \equiv 1$ on $\mathbb{R}^{+}$by Lemma 9. This contradicts assumption (2). We conclude that $\alpha=r(A)$; that is, $\operatorname{Sp}(A)=\{\lambda\}$ for some $\lambda \geqslant 0$. Suppose now that $\lambda<1$. Since $f(1)=1$, we have that $f$ is a constant on the interval $[\lambda, 1]$. Again, Lemma 9 implies that $f \equiv 1$ on $\mathbb{R}^{+}$, a contradiction. Similarly, $\lambda>1$ gives a contradiction. Thus $\lambda=1$, which implies $A=I$.

(2) $\Rightarrow$ (4). Let $A>0$ be such that $A \sigma I=A$. Then $g(A)=A$ where $g$ is the representing function of the transpose of $\sigma$. Hence, $g(x)=x$ for all $x \in \mathrm{Sp}(A)$. Suppose that $\alpha \equiv \inf \operatorname{Sp}(A)<r(A)$. Then $g(x)=x$ for all $x \in$ $[\alpha, r(A)]$. It follows that $g(x)=x$ on $\mathbb{R}^{+}$by Lemma 10 . Hence, the transpose of $\sigma$ is the right-trivial mean. This contradicts assumption (2). We conclude that $\alpha=r(A)$; that is, $\operatorname{Sp}(A)=$ $\{\lambda\}$ for some $\lambda \geqslant 0$. The same argument as in $(2) \Rightarrow(3)$ yields $A=I$.

$(3) \Rightarrow(5)$. Use the congruence invariance of $\sigma$.

$(2) \Rightarrow(6)$. Assume that $\sigma$ is not the left-trivial mean. Let $A \geqslant 0$ be such that $I \sigma A \leqslant I$. Then $f(A) \geqslant I$. The spectral mapping theorem implies that $f(\lambda) \geqslant 1$ for all $\lambda \in \operatorname{Sp}(A)$. Suppose that there exists $t \in \mathrm{Sp}(A)$ such that $t<1$. Since $f(t) \leqslant f(1)=1$, we have $f(t)=1$. It follows that $f(x)=1$ for $t \leqslant x \leqslant 1$. By Lemma $9, f \equiv 1$ on $\mathbb{R}^{+}$, a contradiction. We conclude that $\lambda \geqslant 1$ for all $\lambda \in \operatorname{Sp}(A)$; that is, $A \geqslant I$.

$(2) \Rightarrow(7)$. It is similar to (2) $\Rightarrow(6)$.

$(6) \Rightarrow(8)$. Assume (6). Let $A>0$ be such that $A \leqslant A \sigma I$. Then

$$
A \leqslant A^{1 / 2}\left(I \sigma A^{-1}\right) A^{1 / 2}
$$

which implies $I \leqslant I \sigma A^{-1}$. By (6), we have $\mathrm{I} \leqslant A^{-1}$ or $A \leqslant I$.

$(7) \Rightarrow(9)$. It is similar to $(6) \Rightarrow(8)$.

$(6) \Rightarrow(10)$. Use the congruence invariance of $\sigma$.

$(7) \Rightarrow(11)$. Use the congruence invariance of $\sigma$.

$(2) \Leftrightarrow(12) \Leftrightarrow(16)$. Note that the representing function of the left-trivial mean is the constant function $f \equiv 1$. Its associated measure is the Dirac measure at 0 .

$(2) \Rightarrow(13)$. Assume (2). Let $x \geqslant 0$ be such that $f(x)=1$. Suppose that $x \neq 1$. It follows that $f(x)=1$ for all $x$ lying between $x$ and 1 . Lemma 9 implies that $f \equiv 1$ on $\mathbb{R}^{+}$, contradicting assumption (2). 

(13).

$(2) \Rightarrow(14),(15)$. Modify the argument in the proof $(2) \Rightarrow$

Theorem 12. Let $\sigma$ be a mean with representing function $f$ and associated measure $\mu$. Then the following statements are equivalent:

(1) $\sigma$ is strict at the right;

(2) $\sigma$ is not the right-trivial mean;

(3) for all $A \geqslant 0, A \sigma I=I \Rightarrow A=I$;

(4) for all $A>0, I \sigma A=A \Rightarrow A=I$;

(5) for all $A \geqslant 0$ and $B>0, A \sigma B=B \Rightarrow A=B$;

(6) for all $A \geqslant 0, I \leqslant A \sigma I \Rightarrow I \leqslant A$;

(7) for all $A \geqslant 0, A \sigma I \leqslant I \Rightarrow A \leqslant I$;

(8) for all $A>0, A \leqslant I \sigma A \Rightarrow A \leqslant I$;

(9) for all $A>0, I \sigma A \leqslant A \Rightarrow I \leqslant A$;

(10) for all $A \geqslant 0$ and $B>0, B \leqslant A \sigma B \Rightarrow B \leqslant A$;

(11) for all $A \geqslant 0$ and $B>0, A \sigma B \leqslant B \Rightarrow A \leqslant B$;

(12) $f$ is not the identity function $x \mapsto x$;

(13) $\mu$ is not the associated measure at 1.

Proof. Replace $\sigma$ by its transpose in the previous theorem.

We immediately get the following corollaries.

Corollary 13. A mean is strict if and only if it is nontrivial.

Corollary 14. Let $\sigma$ be a nontrivial mean. For each $A>0$ and $B>0$, the following statements are equivalent:
(i) $A=B$;
(ii) $A \sigma B=A$;
(iii) $A \sigma B=B$;
(iv) $B \sigma A=A$;
(v) $B \sigma A=B$.

The next result is a generalization of [10, Theorem 4.7] in which the mean $\sigma$ is the geometric mean.

Corollary 15. Let $\sigma$ be a nontrivial mean. For each $A>0$ and $B>0$, the following statements are equivalent:
(i) $A \leqslant B$;
(ii) $A \leqslant A \sigma B$;
(iii) $A \sigma B \leqslant B$;
(iv) $A \leqslant B \sigma A$;
(v) $B \sigma A \leqslant B$.

Remark 16. (i) It is not true that if $\sigma$ is not the left-trivial mean then, for all $A \geqslant 0$ and $B \geqslant 0, A \sigma B=A \Rightarrow A=B$. Indeed, take $\sigma$ to be the geometric mean, $A=0$, and

$$
B=\left(\begin{array}{ll}
0 & 0 \\
0 & 1
\end{array}\right) \text {. }
$$

(ii) The assumption of invertibility of $A$ or $B$ in Corollary 14 cannot be omitted, as a counter example in (i) shows. Also, the invertibility of $A$ or $B$ in Corollary 15 cannot be omitted. Consider the geometric mean and

$$
\begin{aligned}
& A=\left(\begin{array}{ll}
1 & 0 \\
0 & 0
\end{array}\right), \\
& B=\left(\begin{array}{ll}
0 & 0 \\
0 & 1
\end{array}\right) .
\end{aligned}
$$

\section{Conflict of Interests}

The author declares that there is no conflict of interests regarding the publication of this paper.

\section{Acknowledgment}

The author is supported by King Mongkut's Institute of Technology Ladkrabang Research Fund Grant no. KREF045710.

\section{References}

[1] G. Toader and S. Toader, Greek means and the arithmeticgeometric mean [dissertation], Victoria University, 2005.

[2] J. Anderson and R. J. Duffin, "Series and parallel addition of matrices," Journal of Mathematical Analysis and Applications, vol. 26, pp. 576-594, 1969.

[3] F. Kubo and T. Ando, "Means of positive linear operators," Mathematische Annalen, vol. 246, no. 3, pp. 205-224, 1979/80.

[4] F. Hiai, "Matrix analysis: matrix monotone functions, matrix means, and majorization," Interdisciplinary Information Sciences, vol. 16, no. 2, pp. 139-248, 2010.

[5] P. Chansangiam and W. Lewkeeratiyutkul, "Characterizations of connections for positive operators," Southeast Asian Bulletin of Mathematics, vol. 37, no. 5, pp. 645-657, 2013.

[6] K. Löwner, "Über monotone matrixfunktionen," Mathematische Zeitschrift, vol. 38, no. 1, pp. 177-216, 1934.

[7] R. Bhatia, Matrix analysis, vol. 169 of Graduate Texts in Mathematics, Springer, New York, NY, USA, 1997.

[8] F. Hiai and K. Yanagi, Hilbert Spaces and Linear Operators, Makino, 1995.

[9] F. Hansen and G. K. Pedersen, "Jensen's inequality for operators and Löwner's theorem," Mathematische Annalen, vol. 258, no. 3, pp. 229-241, 1982.

[10] M. Fiedler and V. Pták, "A new positive definite geometric mean of two positive definite matrices," Linear Algebra and Its Applications, vol. 251, pp. 1-20, 1997.

The case of right-trivial mean is just the same. 


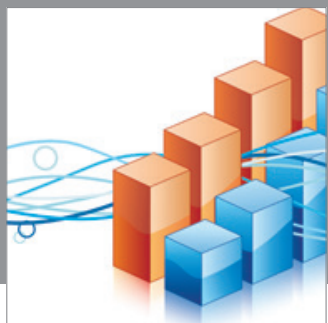

Advances in

Operations Research

mansans

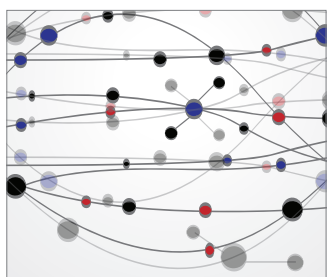

The Scientific World Journal
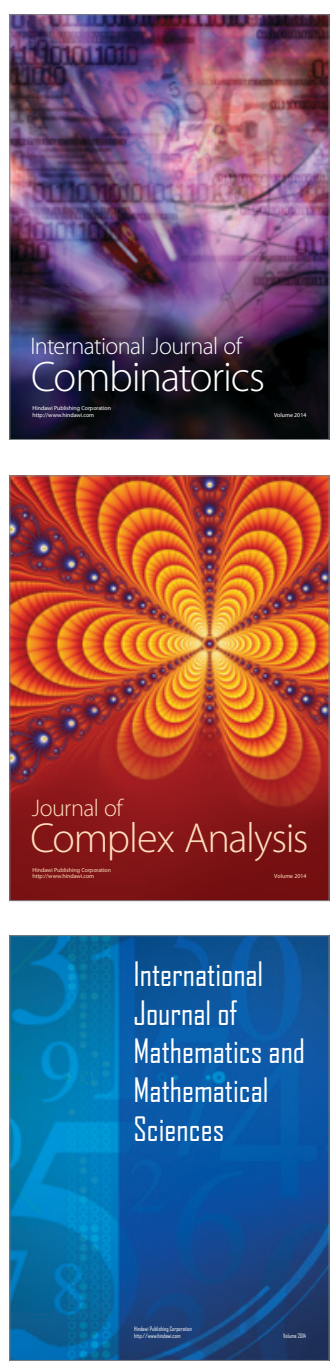
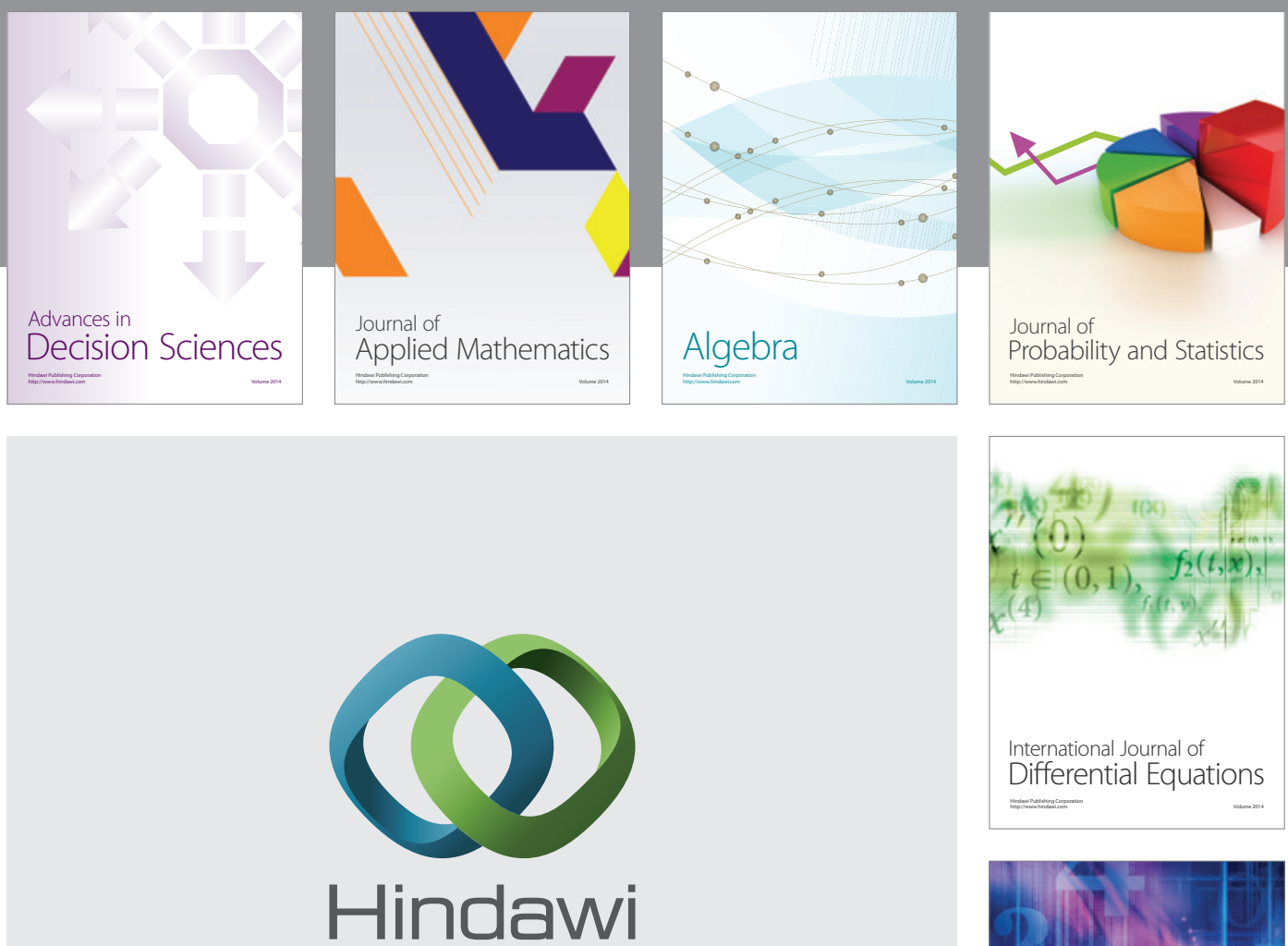

Submit your manuscripts at http://www.hindawi.com
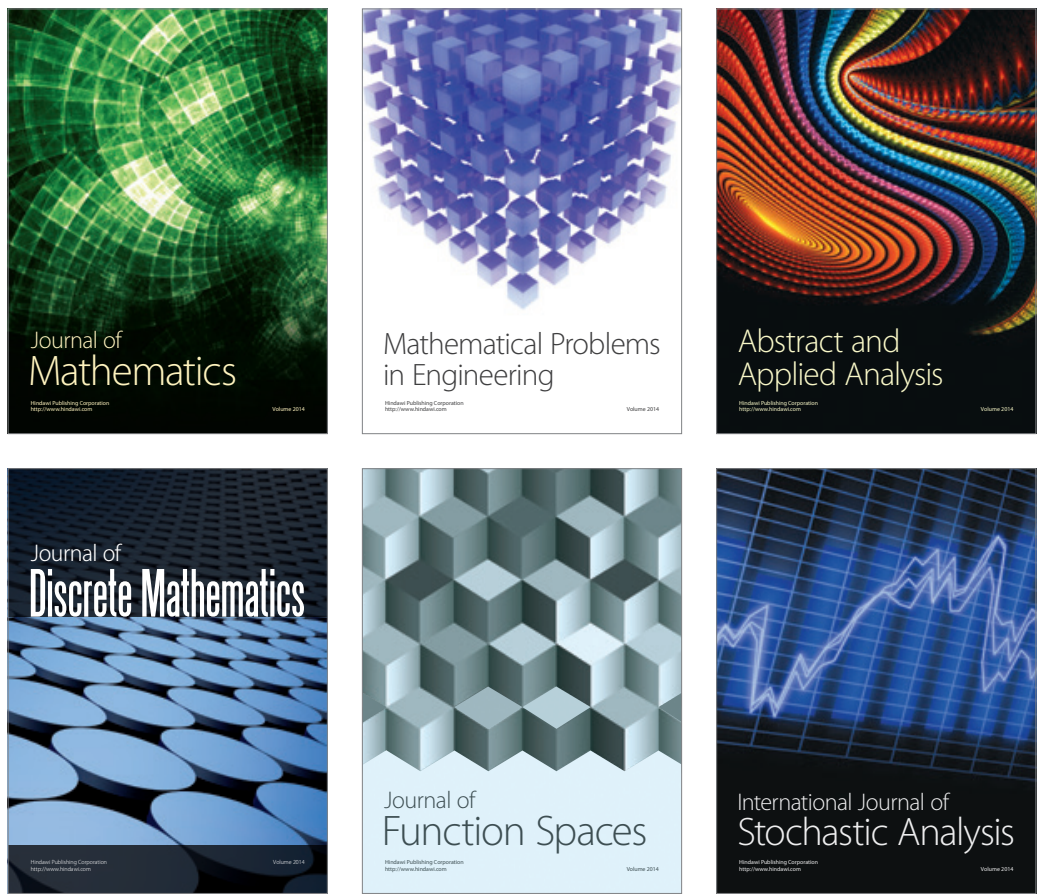

Journal of

Function Spaces

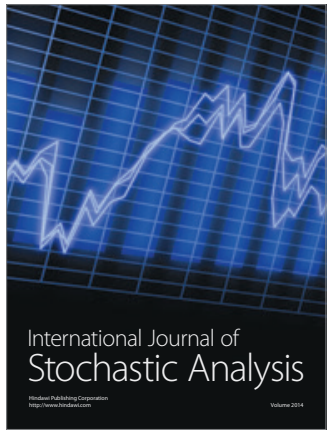

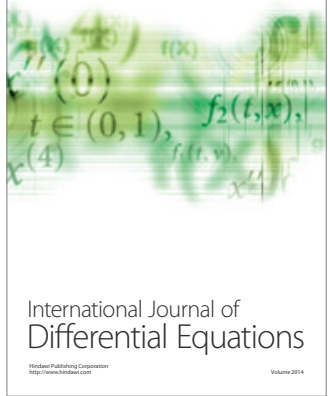
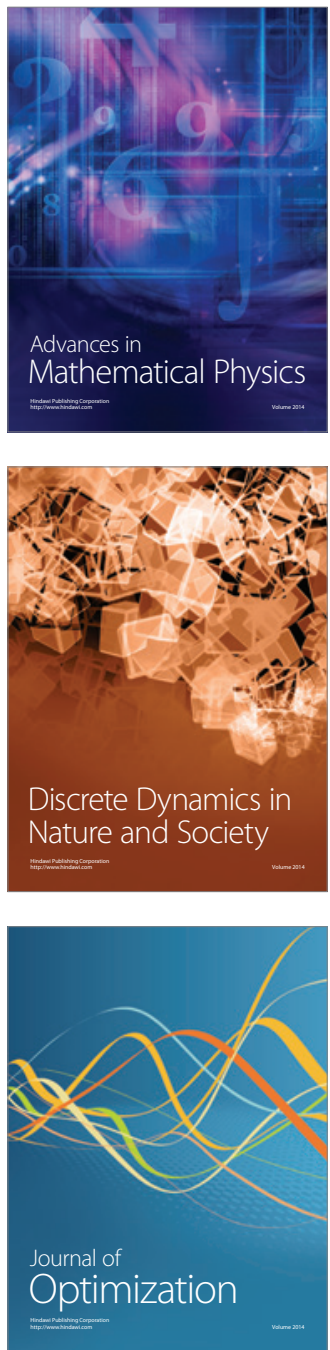